\title{
Efficacy of Mentalization-Based Group Therapy for Adolescents: A Pilot Randomised Controlled Trial
}

\author{
Helen Griffiths ${ }^{1,2^{*}}$, Fiona Duffy ${ }^{1,2}$, Louise Duffy ${ }^{2}$, Sarah Brown ${ }^{1,2}$, Harriet Hockaday ${ }^{1,2}$, \\ Emma Eliasson $^{1,2}$, Jessica Graham ${ }^{2}$, Alice Thompson ${ }^{1,2}$, Rachel Happer ${ }^{2}$, Maeve Butler ${ }^{1,2}$, \\ Matthias Schwannauer ${ }^{1,2}$ \\ ${ }^{1}$ Department of Clinical Psychology, School of Health in Social Science, The University of \\ Edinburgh, Medical School (Doorway 6), Teviot Place, EH8 9AG \\ ${ }^{2}$ NHS Lothian Child and Adolescent Mental Health Services, Royal Edinburgh Hospital, Tipperlinn \\ Road, Edinburgh, EH10 5HF
}

\begin{abstract}
Background: Suicide is the leading cause of death in adolescents. Furthermore, up to one quarter of adolescents who self-harm will repeat self-harm within one year, highlighting the need for evidence-based prevention and treatment services. Mentalization Based Therapy (MBT) has yielded promising outcomes for individuals who self-harm, however to date only one study has examined MBT in adolescents, wherein the treatment protocol consisted of individual and family therapy. Currently, there has been no development or examination of MBT-A in a group format for adolescents.
\end{abstract}

Methods/Design: The present study is a randomised controlled single blind feasibility trial that aims to (1) adapt the original explicit MBT introductory group manual for an adolescent population (MBT-Ai) and to (2) assess the feasibility of MBT-Ai through examination of consent rates, attendance, attrition and self-harm. Participants are adolescents presenting to Child and Adolescent Mental Health Services (CAMHS) with self-harming behaviors within the last 6 months. Young people will be randomised to a 12-week MBT-Ai group plus treatment as usual (TAU) or TAU alone. Participants will be assessed at baseline and at 12-, 24- and 36-weeks post-baseline.

Discussion: This paper describes the development of a treatment manual and the protocol of a randomised controlled feasibility trial of MBT-Ai aimed at treating adolescents who selfharm. Further investigation of a full-scale trial will be necessary to instill benefits if pilot results suggest efficacy.

Trial registration: NCT02771691

Keywords: adolescent, mentalization, MBT, group, self-harm

* Correspondence to Dr. Helen Griffiths, Department of Clinical Psychology, School of Health in Social Science, The University of Edinburgh, Medical School (Doorway 6), Teviot Place, EH8 9AG. Email: Helen.Griffiths@ed.ac.uk 


\section{Background}

Suicide is a leading cause of death in adolescence worldwide (Centers for Disease Control, 2014; WHO, 2017), resulting in over 67,000 deaths in adolescents aged 10-19 in 2015, or approximately 184 deaths a day (World Health Organization, 2017). Psychiatric history, suicide intent and self-harm are among the strongest risk factors for suicide, with evidence suggesting self-harm increases the risk of death by suicide approximately 10 -fold (Hawton \& Harriss, 2007, Chan et al., 2016). Although true prevalence of self-harm is difficult to depict based on varying definitions, the most well-known cross-sectional study to date documented a life-time prevalence of self-harm to be $27.6 \%$ in adolescents across 11 European countries, highlighting the scope of the problem (Brunner et al., 2014). Furthermore, repetition of selfharm is alarmingly common, and between $10-25 \%$ of adolescents will repeat self-harm within a year (Hawton \& Harriss, 2008; Hawton \& James, 2005)

Self-harm often occurs within the context of a wider, complex mental health presentation, including the interaction of a variety of social, psychological and cultural factors (Hawton, Saunders \& O'Connor, 2012). Personality traits apparent in adult borderline personality disorder (BPD), including emotion dysregulation and decreased social functioning, have been found to be predictive of and common within young people who self-harm (Mikolajczak, Petrides \& Hurry, 2009; Crowell et al., 2012). Individuals with BPD show reduced capacities to mentalize - the imaginative mental activity that enables us to perceive and interpret human behaviour in terms of intentional mental states (e.g., needs, desires, feelings, beliefs, goals, purposes, and reasons) (Allen, Fonagy \& Bateman, 2008; Bateman \& Fonagy, 2006). Mentalizing deficits are linked to problems with emotional regulation and impulsivity, especially within interpersonal interactions. This is frequently associated with significant emotional distress, self-harm and crisis presentations.

There is an urgent need to reduce the high rates of repetition of self-harm seen within adolescents. Appropriate early intervention can often also prevent the escalation of parallel emotional distress and functional impairments. A recent meta-analysis by Ougrin and colleagues (2015) evidenced promising effects for three therapeutic interventions, namely cognitive behavioural therapy, dialectal behaviour therapy, and mentalization-based therapy however, none of these effects had been independently replicated. This emphasizes the need for further examination. Furthermore, there remains a gap between clinical practice and research evidence, whereby best-practice within clinical settings is often based on interventions with little to no evidence (Burns et al., 2005). The need to further develop and replicate trials for self-harm in adolescence has been highlighted throughout the literature (Ougrin et al., 2015; Ougrin et al., 2012; De Silva et al., 2013; Hawton et al., 2015).

Mentalization Based Therapy (MBT) was initially developed for the treatment of adults with a diagnosis of borderline personality disorder (BPD). It is proposed that a fragile mentalizing capacity vulnerable to social and interpersonal interaction is a core feature of the disorder (Bateman \& Fonagy, 2010). Within this adult population, MBT reduced depression, anxiety, suicidal and self-mutilatory acts; and inpatient days, and improved social and interpersonal function in comparison to treatment as usual (Bateman \& Fonagy, 1999) with continued improvements at eighteen months (Bateman \& Fonagy, 2001). MBT has also been shown to be effective in an adult outpatient setting (Bateman \& Fonagy, 2009). These findings require independent replication.

There are very few randomised controlled trials (RCTs) to date which have evaluated MBT for adolescents (MBT-A). Rossouw \& Fonagy (2012) found that MBT-A was more effective than treatment as usual for adolescents who self-harm, with a recovery rate of $44 \%$ in comparison to $17 \%$. The treatment protocol involved weekly individual MBT-A sessions and monthly MBT family therapy (MBT-F). We are aware of one other mentalization-based

Social Science Protocols, April 2018, 1-14. 
therapy trial for adolescents in progress. Beck et al (2016) are investigating a group-based mentalization-based treatment programme for adolescents with BPD or subthreshold BPD. Given that the evidence base for MBT-A is still in its infancy, it is not yet possible to determine which modalities of MBT (individual, group, family) are most effective or instrumental in any observed clinical change.

Our own programme consists of an adaptation of an explicit introductory MBT group with adults (MBTi; Karterud \& Bateman, 2011). One of the aims of this introductory group is the development of MBT knowledge about underlying principles and concepts of mentalization. Whilst MBTi for the adult population is designed as a precursor to a combination of MBT individual and implicit group therapy, our MBT-Ai programme incorporates a number of experiential tasks and role plays that not only provide psychoeducation about mentalization but also encourage the application of mentalization principles to common interpersonal dilemmas. Our adolescent population present to National Health Service (NHS) Child and Adolescent Mental Health Services (CAMHS) with difficulties relating to self-harm behaviours, emotional distress and interpersonal problems. In this paper we describe the development of a novel group-based MBT-Ai programme and the methodology of the present study, a pilot RCT comparing MBT-Ai plus treatment as usual (TAU) to TAU alone.

\section{Methods/Design}

\subsection{Aims and hypotheses}

The present study aims to evaluate the feasibly and effects of 12 week MBT-Ai for the reduction of self-harm and crisis presentations in a group of young people already receiving treatment within specialist NHS Child and Adolescent Mental Health Services. The following study methodology will be reported in accordance with the Standard Protocol Items: Recommendations for Interventional Trials (SPIRIT) statement and guidelines (Chan et al., 2013). See figure 1.

\section{Primary research questions:}

1. What proportion of potential participants consent to participate in a randomised controlled trial comparing Mentalization Based Treatment for Adolescents delivered as group therapy plus treatment as usual to treatment as usual alone?

2. Does Mentalization Based Treatment for Adolescents delivered as group therapy provide an acceptable treatment option for adolescents who self-harm?

\section{Secondary research questions:}

1. What are the clinical characteristics of this group at entry to the study?

2. What are the clinical characteristics of this group at the end of intervention and at follow-up?

3. Does receipt of Mentalization Based Treatment for Adolescents delivered as group therapy plus treatment as usual lead to reduced self-harm and crisis presentations compared to treatment as usual alone?

4. Are the study procedures appropriate and acceptable?

\subsection{Study design}

This study is a two-arm, single (rater) blind, randomised controlled trial registered with ClinicalTrials.gov (Trial registration: NCT02771691). 
Figure 1. SPIRIT recommended content for the schedule of enrolment, interventions, and assessments.

\begin{tabular}{|c|c|c|c|c|c|c|c|c|c|}
\hline \multirow[b]{3}{*}{ TIMEPOINT $^{\star *}$} & \multirow{3}{*}{$\begin{array}{c}\text { Enrolment } \\
-t_{1}\end{array}$} & \multicolumn{8}{|c|}{ STUDY PERIOD } \\
\hline & & \multirow{2}{*}{$\begin{array}{c}\text { Allocation } \\
0\end{array}$} & \multicolumn{5}{|c|}{ Post-allocation } & \multirow[b]{2}{*}{$t_{6}$} & \multirow{2}{*}{$\begin{array}{c}\text { Close-out } \\
t_{x}\end{array}$} \\
\hline & & & $t_{1}$ & $t_{2}$ & $t_{s}$ & $t_{4}$ & $t_{5}$ & & \\
\hline \multicolumn{10}{|l|}{ ENROLMENT: } \\
\hline \multirow{2}{*}{$\begin{array}{l}\text { Eligibility screen } \\
\text { Informed consent }\end{array}$} & $\mathrm{X}$ & & & & & & & & \\
\hline & $\mathrm{X}$ & & & & & & & & \\
\hline Allocation & & $\mathrm{X}$ & & & & & & & \\
\hline \multicolumn{10}{|l|}{ INTERVENTIONS: } \\
\hline \multicolumn{10}{|l|}{ MBT-Ai } \\
\hline \multicolumn{10}{|l|}{ TAU } \\
\hline \multicolumn{10}{|l|}{ ASSESSMENTS: } \\
\hline \multirow{2}{*}{ RTSHIA } & & $\mathrm{X}$ & & $\mathrm{X}$ & & $\mathrm{X}$ & & $\mathrm{X}$ & \\
\hline & & $\mathrm{x}$ & & $x$ & & $\mathrm{x}$ & & $\mathrm{X}$ & \\
\hline RCADS & & $\mathrm{x}$ & $x$ & $x$ & $x$ & $\mathrm{x}$ & $x$ & $x$ & \\
\hline \multirow{2}{*}{ DERS } & & $x$ & & $\mathrm{x}$ & & $\mathrm{x}$ & & $x$ & \\
\hline & & $x$ & & $\mathrm{x}$ & & $\mathrm{X}$ & & $\mathrm{X}$ & \\
\hline ISM & & $\mathrm{x}$ & & $\mathrm{X}$ & & $\mathrm{x}$ & & $\mathrm{X}$ & \\
\hline BPFSC & & $x$ & & $x$ & & $\mathrm{x}$ & & $\mathrm{x}$ & \\
\hline $\begin{array}{c}\text { Demographics + } \\
\text { SH incidents }\end{array}$ & & & & & & & & & $x$ \\
\hline
\end{tabular}

Note: $\mathrm{SH}=$ self-harm

$* * \mathrm{t}_{1}=6$ weeks; $\mathrm{t}_{2}=12$ weeks; $\mathrm{t}_{3}=18$ weeks; $\mathrm{t}_{4}=24$ weeks; $\mathrm{t}_{5}=30$ weeks; $\mathrm{t}_{6}=36$ weeks. 


\subsection{Setting}

The project involves a collaboration between NHS Lothian CAMHS clinicians and the University of Edinburgh. NHS Lothian CAMHS serves a population of approximately 160,000 young people under 18 years and provides a range of outpatient and more specialist services. Staff from non-inpatient Tier 4 services, which include day programmes and assertive outreach teams, have received training in Adolescent Mentalization-Based Integrative Treatment (AMBIT) (Bevington et al., 2013). Four trained MBT therapists from this service developed and piloted the MBT-Ai group manual under supervision provided by the Anna Freud Centre.

\subsection{Participants}

Up to sixty adolescent participants will be recruited from NHS Lothian CAMHS. Inclusion criteria are as follows: (1) Aged 12-18 years (2) self-harm behaviour in the past 6 months (3) in receipt of treatment from NHS Lothian CAMHS (4) competent and willing to provide written, informed consent. Participants will be excluded based on the following criteria: (1) severe learning disability or pervasive developmental disorder (2) acute psychotic episode (3) eating disorder in the absence of self-harm (4) non-English speaking (5) current involvement in other ongoing treatment research.

The sample size of $n=60$ was deemed achievable given previous referral rates into the pilot stages of the project and the number of young people within CAMHS who present with selfharm behavior. Allowing for likely attrition, this sample size will give us sufficient data in order to report on response rates, follow-up rates, safety information and attrition, as well as the clinical characteristics of our study population at the beginning and end of the trial and follow-up. Please see figure 2. 
Figure 2. Participant/study flow chart

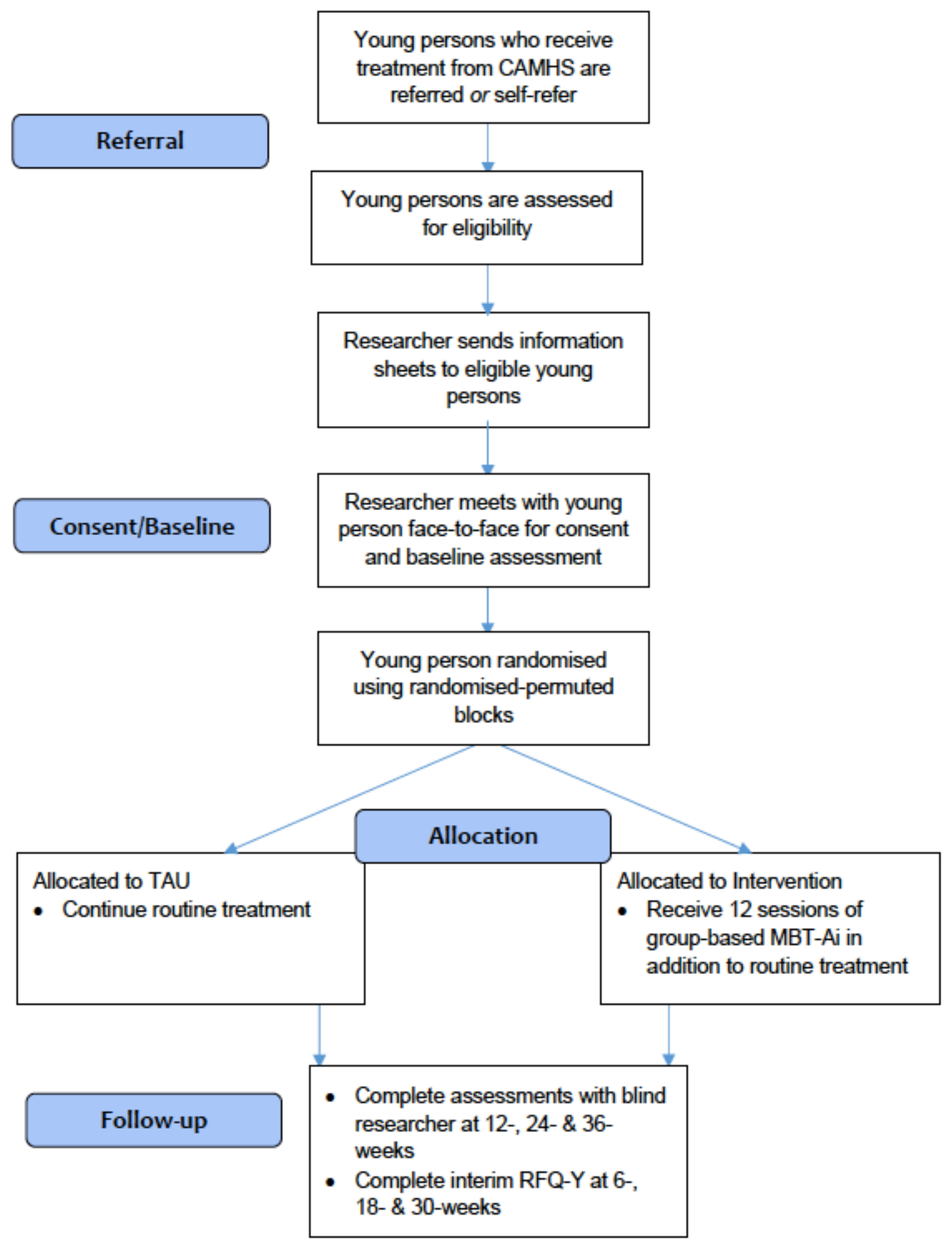

\subsection{Procedure}

The research assistants will be independent, blind assessors who will conduct all assessments of primary and secondary outcomes. Operationally, the Research Assistants (RAs) will liaise regularly with the relevant clinical teams. Key clinicians from the teams 
(e.g. psychiatrists, community mental health workers, psychologists, etc.) will be given information about the study and asked to identify young people they consider to meet the inclusion/exclusion criteria. The clinician will be asked to tell the young person about the study, offer them a participant information sheet and ask them if they want to consider taking part. Self-referrals will also be accepted for those who wish to participate in the trial and who have been informed of the trial via the trial poster or client leaflet. If any young person selfrefers to the trial, they will be informed that the researcher will need to contact their clinical care team to inform them of their interest in the trial and to check details regarding inclusion/exclusion criteria. The treating clinician must also confirm that the young person has capacity to consent.

If a young person expresses interest in the trial, they will be provided with a Participant Information Sheet and given at least 24 hours to consider their involvement. The research assistant will then contact the young person and, if they wish to participate, arrange an appointment with them in order to discuss any queries before, if appropriate, obtaining the participant's consent. We will apply the principle of direct consent for all potential participants. For those under the age of 14 years, we will provide an information sheet for parents/guardians. During the recruitment/consenting process the researcher will ensure that the young person is fully informed of the randomization process and their chances of receiving MBT-Ai group therapy.

Once informed and written consent has been obtained, baseline measures will be completed. Measures will be completed within a single 30-minute session at each time point wherever possible. Primary and secondary outcomes will be carried out at baseline and 12, 24 and 36 weeks. Assessment of putative mechanisms will additionally take place at 6 week intervals. Case note review will be completed at the end of treatment.

Participants will be withdrawn from the trial if they withdraw consent. A distinction will be made as to whether the individual is withdrawing consent from further trial treatment only or withdrawing from trial treatment and follow-up. Drop-out rates to each arm will be reported.

\subsection{Randomisation/treatment allocation}

Randomisation (at the individual level) will be independent and concealed, using randomised-permuted blocks adjusted to permit access to group treatment without undue delay. The Research Assistants evaluating baseline and the study outcomes will be blind to randomized allocation. Masking will be maintained using a wide range of measures. All trial unblindings will be reported to the Trial Manager who will implement corrective action if necessary. Group allocation will remain concealed until completion of the ratings.

Following randomization, the allocation will be made known to the participant, their clinical team, and the trial therapist (as appropriate). If the participant is randomised to MBTAi plus TAU then the participant will meet briefly with a qualified therapist for orientation to the MBT-Ai group programme. They will be offered 12-24 sessions of group-based MBT-Ai on a weekly basis. If a participant is randomised to TAU, then they will receive only TAU. Referrers will not be asked to withhold any treatment as this was considered to be unethical. Both conditions will receive an assessment session conducted by a researcher who is blind and independent to the treatment group at baseline, 12 weeks, 24 weeks and 36 weeks. A further self-report questionnaire (the Reflective Functioning Questionnaire for Youths; Ha et al., 2013) will be additionally administered at 6 weeks, 18 weeks and 30 weeks in order to measure potential changes in young people's capacity to mentalize.

With the participant's consent, further information will be collated from case note review/routinely collected information held in NHS Lothian electronic patient records. The

Social Science Protocols, April 2018, 1-14. 
information will be used to collect additional information about any potential self-harm behaviour or crisis presentations to the NHS as well as to contextualize the TAU package through populating the relevant sections of the Client Service Receipt Inventory (CSRI; Beecham \& Knapp, 1992) which will be adapted for use with our adolescent population. The relevant sections of the CSRI will provide a standardized measure of health services accessed by the young people over the study period. Other variables to be recorded include gender, age, number of treatment sessions received, service engagement and dropout rate.

\subsection{Study arms}

\section{a) Mentalization Based Treatment for Adolescents (MBT-Ai) group therapy}

Up to 10 young people will attend each group. The active treatment will be delivered by appropriately qualified MBT-A trial therapists under the supervision of an MBT accredited supervisor. Fidelity to protocol and adherence to the principles of MBT will be monitored during supervision and checked by means of audiotape rated by an experienced MBT clinician independent of the delivery of either intervention (subject to participants consenting to sessions being taped. Failure to consent to taped sessions will not affect participation in the intervention).

The MBT-Ai group programme bases its foundations in the MBTi adult manual (Karterud \& Bateman, 2011). The adult MBTi model consists of twelve weekly 1.5-hour group sessions facilitated by two therapists with 6-12 members. Adult MBTi sessions include an introduction to the concept of mentalization, emotional literacy, attachment relationships, information on personality disorders, anxiety and depression, and an MBT treatment programme overview. Clinicians from NHS Lothian Tier IV services (designed for complex/severe presentations where more intensive input is required) are trained in Adolescent Mentalization Based Integrative Therapy (AMBIT; Bevington et al., 2013) and therefore use mentalization based principles and interventions in their therapeutic work. The MBT-Ai group was therefore designed to either accompany other mentalization based interventions or to act as a standalone mentalization group programme. The target population was adolescents displaying impulsive self-harming. This group of young people was also likely to experience interpersonal difficulties and problems with emotional dysregulation.

MBT-Ai takes place over twelve sessions facilitated by two MBT trained clinicians with up to ten participants in each group. The group aims are:

- Introduce concepts of mentalization, attachment and encourage emotional literacy.

- Encourage reflection on interpersonal relationship patterns and their development.

- Reflect on how these concepts effect emotional expression, behaviour and mental health.

- Prepare young people for longer-term therapeutic relationships.

- $\quad$ Elicit more detail about individual mentalizing capacity.

Significant modifications to the original adult manual were made to omit descriptions of an MBT programme and the psychoeducation sessions on personality disorders. Adaptations were also made to make it more acceptable and engaging to an adolescent population, to provide greater experiential content and to encourage the application of mentalization techniques to common daily dilemmas. Changes included:

- Shorter sessions: MBTi suggests 1.5 hour sessions however, in our experience, adolescents' ability to tolerate long group sessions is reduced, therefore MBT-Ai sessions were limited to 1.25 hours.

- Interactive techniques (warm up games, worksheets, video clips etc.) to support participation and promote learning

- Simplified language and more sessions on complex concepts

Social Science Protocols, April 2018, 1-14. 
- More sessions on emotional literacy to consolidate basic emotional language before progressing to the more complex concept of emotion regulation.

Following service user feedback elicited during a focus group during the development phase of MBT-Ai, significant modifications were made to the MBT-Ai manual including reducing the written content of handouts, increasing the number of interactive exercises, and closing the group after three missed sessions to improve group cohesion and participation.

\section{b) Treatment as Usual (TAU)}

Participants randomized to TAU will receive treatment as usual from NHS Lothian CAMHS. It is expected that TAU will consist of a combination of key worker input, psychological/psychosocial intervention and medication. TAU will be delivered according to national and local service protocols and guidelines and will not be standardised for the purposes of the research. In order to establish the parameters of the TAU package, service use will be measured using an adapted version of the Client Service Receipt Inventory (Beecham \& Knapp, 1992).

\section{Data Collection}

\subsection{Outcome measures}

In order to address the primary research questions, the main outcomes will be the proportion of young people who consent to randomisation, and attendance and attrition rates monitored by group facilitators on a weekly basis.

In order to answer the secondary research questions, the outcome measures will be as follows:

- Self-harm and risk-taking as measured by the self-harm and risk-taking subscales of the Risk-Taking and Self-Harm Inventory (RTSHI; Vrouva et al., 2010). This is a self-report measure designed to assess adolescent risk-taking and self-harm in community and clinical settings. In the original study, both the self-harm and risktaking factors of the RTSHI have evidenced high internal consistency, test-retest reliability and sufficient validity in youth.

- Self-harm related hospital use as reported in clinical records and/or via NHS Lothian electronic patient records

- Emotional distress (anxiety and depression) as measured by the Revised Child Anxiety and Depression Scale (RCADS; Chorpita et al., 2000). RCADS has evidenced reliability and validity in both clinical (Chorpita, Moffitt \& Gray, 2005) school-based samples of children and adolescents (Van Oort et al., 2009; Piqueras et al., 2007).

- Mentalization as measured by the self-report Reflective Functioning Questionnaire for Youths (RFQ-Y; Ha et al., 2013). This measure has evidenced adequate internal consistency, convergent validity and reliability in adolescent samples.

- Emotion regulation as measured by the Difficulties in Emotion Regulation Scale (DERS; Gratz \& Roemer, 2004). This measure has evidenced high internal consistency, adequate validity and good test-retest reliability in young adults (Gratz \& Roemer, 2004) and adolescents (Weinberg \& Klonsky, 2009).

- Interpersonal sensitivity as measured by the Interpersonal Sensitivity Measure (ISM; Boyce \& Parker, 1989). Validity and reliability has been evidenced in populations diagnosed with depression and anxiety (Boyce \& Parker, 1989; Harb et al., 2002).

- Borderline Traits as measured by the 11-item short version of the Borderline Personality Features Scale for Children (BPFSC; Sharp et al., 2014). In the original 
study, satisfactory construct and criterion validity in adolescent samples were established.

- Attachment as measured by the 12-item short version of the Experiences in Close Relationships Scale-Revised Child version (ECRS-RC; Brenning et al., 2014). This instrument has shown excellent validity and reliability in youth samples (Brenning et al., 2014).

All measures will be administered at baseline, 12, 24 and 36 weeks. In addition, mentalization as assessed by the RFQ-Y will be additionally taken at 6,18 and 30 weeks. All measures will be carried out by the Research Assistants who will be graduates with a good undergraduate degree in Psychology. The RA will be trained by chief investigator/principal investigator and receive weekly supervision on all aspects of their role.

\subsection{Statistical analysis}

As a feasibility study, the majority of our reporting will be descriptive. However, in order to assess whether the receipt of MBT-Ai plus TAU compared to TAU alone leads to reduced self-harm and crisis presentations we will also carry out intention-to-treat analyses to determine treatment effects on the primary and secondary outcome measures, adjusting for pre-specified baseline covariates. An exploratory repeated measures regression model, adjusting for baseline measures (including age, gender, and the baseline measure of the outcome of interest) will be fitted to assess the treatment effects on the primary and secondary outcome measures and the evolution of these treatment effects over time. We will also explore subscales of the outcome measures to see if there is strong treatment signals within these sub scales. Exploratory linear multiple regressions, in which all predictors and covariates are entered independently into the regression algorithm to avoid any artificial inflation of estimated $\mathrm{R}^{2}$, will be adopted to investigate the mediational effects of the putative underlying mechanisms of change. We will also examine the effect of number of sessions received as a putative moderator.

\subsection{Ethics approvals}

Ethical approval was received from the South East Scotland Research Ethics committee (REC No: 15/SS/0142). Consent to participate will be required prior to participation in this study.

\section{Discussion}

This paper describes the development of a group-based MBT-Ai and the protocol for a RCT pilot that aims to compare MBT-Ai plus TAU to TAU alone on the reduction of selfharm in a Child and Adolescent Mental Health Service. Mentalisation-based therapy has shown promising effects (Rossouw \& Fonagy, 2012) in adolescents who self-harm, however to date only this single trial of MBT-A has been completed. Furthermore, there is currently no evidence for group-based MBT-Ai. It is not known which MBT modality (individual, group, family) is most effective, nor how long therapy needs to last in order to confer benefit. As we know that clinical CAMH services are stretched for resources, comparatively brief, group-based MBT-Ai could potentially increase access to treatment for young people who self-harm, translating into feasible practice. Furthermore, by employing a multiple-time point data methodology, we will be able to examine when and if MBT-Ai yields changes related to risk-taking, self-harm, distress and interpersonal outcomes in young people who self-harm. If MBT-Ai shows signals that the intervention is potentially beneficial in this population, the 
researchers will seek further funding to continue protocol development and conduct a largerscale RCT in order to confirm efficacy.

\section{List of Abbreviations}

$\begin{array}{ll}\text { AMBIT } & \text { Adolescent Mentalization-Based Integrative Treatment } \\ \text { BPD } & \text { Borderline Personality Disorder } \\ \text { BPFSC } & \text { Borderline Personality Features Scale for Children } \\ \text { CAMHS } & \text { Child and Adolescent Mental Health Services } \\ \text { CAMH } & \text { Child and Adolescent Mental Health } \\ \text { CSRI } & \text { Client Service Receipt Inventory } \\ \text { DERS } & \text { Difficulties in Emotion Regulation Scale } \\ \text { ECRS-RC } & \text { Experiences in Close Relationships Scale-Revised Child version } \\ \text { ISM } & \text { Interpersonal Sensitivity Measure } \\ \text { MBT } & \text { Mentalization Based Therapy } \\ \text { MBT-A } & \text { Mentalization Based Treatment for Adolescents } \\ \text { MBT-Ai } & \text { Mentalization Based Treatment for Adolescents - Group format } \\ \text { MBT-F } & \text { Mentalization Based Treatment - Family Therapy } \\ \text { MBTi } & \text { Mentalization Based Treatment - Introductory } \\ \text { NHS } & \text { National Health Service } \\ \text { RA } & \text { Research Assistant } \\ \text { RCADS } & \text { Revised Child Anxiety and Depression Scale } \\ \text { RCT } & \text { Randomized Controlled Trial } \\ \text { RFQ-Y } & \text { Reflective Functioning Questionnaire for Youths } \\ \text { RTSHI } & \text { Risk-Taking and Self-Harm Inventory } \\ \text { SPIRIT } & \text { Standard Protocol Items: Recommendations for Interventional Trials } \\ \text { TAU } & \text { Treatment as Usual }\end{array}$

\section{Declarations}

Availability of data and material: The datasets used and/or analysed during the current study are available from the corresponding author on reasonable request.

Competing interests: The authors declare that they have no competing interests.

Funding: Funding was received from the Edinburgh and Lothian's Health Foundation. The funding body had no role in study design, collection, analysis and interpretation of data.

Authors' contributions: MS, HG, and FD developed the study proposal and act as principal investigators. JG, $\mathrm{HH}, \mathrm{EE}, \mathrm{AT}, \mathrm{MB}$ and $\mathrm{SB}$ are all research assistants on the project who contributed to planning data collection and recruitment and assessing feasibility of data collection. FD, LD, RH and HG all had a role in the manual development and delivery of the MBT-Ai group programme. HG, FD and SB were major contributors in writing the manuscript. All authors read and approved the final manuscript.

Acknowledgements: This research is supported by an Edinburgh and Lothians Health Foundation grant to CAMHS Psychology, NHS Lothian (ELHF10-595). The authors would 
also like to acknowledge the supervision of Professor Anthony Bateman to support the development of the group manual.

\section{References}

Allen, J.G., Fonagy, P., \& Bateman, A. (2008). Mentalizing in Clinical Practice. American Psychiatric Publishing, Inc.

Bateman, A., \& Fonagy, P. (1999). Effectiveness of partial hospitalization in the treatment of borderline personality disorder: A randomized controlled trial. American Journal of Psychiatry, 156(10), 1563-1569. doi: 10.1176/ajp.156.10.1563

Bateman, A., \& Fonagy, P. (2001). Treatment of borderline personality disorder with psychoanalytically oriented partial hospitalization: an 18-month follow-up. American Journal of Psychiatry, 158(1), 36-42. doi: 10.1176/appi.ajp.158.1.36

Bateman, A. W., \& Fonagy, P. (2006). Mentalization based treatment for borderline personality disorder: A practical guide. Oxford: Oxford University Press

Bateman, A. W. \& Fonagy, P. (2009). Randomized controlled trial of outpatient mentalization-based treatment versus structured clinical management for borderline personality disorder. The American Journal of Psychiatry, 166(12), 1355-1364.

Bateman, A., \& Fonagy, P. (2010). Mentalization based treatment for borderline personality disorder. World Psychiatry, 9(1), 11-15. doi: 10.1002/j.2051-5545.2010.tb00255.x

Beecham, J.K., \& Knapp, M.R.J. (1992). Costing psychiatric interventions. In G. Thornicroft, C. Brewin, J.K. Wing (Eds.), Measuring mental health needs (pp. 163-183). London: Gaskell.

Beck, E., Bo, S., Gondan, M., Poulsen, S., Pedersen, L., Pedersen, J., \& Simonsen, E. (2016). Mentalization-based treatment in groups for adolescents with borderline personality disorder (BPD) or subthreshold BPD versus treatment as usual (M-GAB): Study protocol for a randomized controlled trial. Trials, 17(1). doi: 10.1186/s13063-016-1431-0

Bevington, D., Fuggle, P., Fonagy, P., Target, M., \& Asen, E. (2013). Innovations in practice: Adolescent mentalization-based integrative therapy (AMBIT ) - a new integrated approach to working with the most hard to reach adolescents with severe complex mental health needs. Child and Adolescent Mental Health, 18(1), 46-51. doi: 10.1111/j.14753588.2012.00666.x

Boyce, P. \& Parker, G. (1989). Development of a scale to measure interpersonal sensitivity. Australian and New Zealand Journal of Psychiatry, 23(3), 341-351.

Brenning, K., Van Petegem, S., Vanhalst, J., \& Soenens, B. (2014). The psychometric qualities of a short version of the Experiences in Close Relationships Scale - Revised Child version. Personality and Individual Differences, 68, 118-123. doi: 10.1016/j.paid.2014.04.005

Brunner, R., Kaess, M., Parzer, P., Fischer, G., Carli, V., Hoven, C.W., . . Wasserman, D. (2014). Life-time prevalence and psychosocial correlates of adolescent direct self-injurious behavior: A comparative study of findings in 11 European countries. Journal of Child Psychology and Psychiatry, 55(4), 337-348. doi: 10.1111/jcpp.12166

Burns, J., Dudley, M., Hazell, P., \& Patton, G. (2005). Clinical management of deliberate self-harm in young people: The need for evidence-based approaches to reduce repetition. Australian and New Zealand Journal of Psychiatry, 39(3), 121-128,39(3), 121128. doi: 10.1080/j.1440-1614.2005.01532.x

Centers for Disease Control and Prevention. (2014). 10 Leading causes of death by age group, United States. Retrieved from https://www.cdc.gov/injury/images/lccharts/leading_causes_of_death_age_group_2014_1 050w760h.gif.

Social Science Protocols, April 2018, 1-14.

http://dx.doi.org/10.7565/ssp.2018.2647 
Chan, M., Bhatti, H., Meader, N., Stockton, S., Evans, J., O'Connor, R., . . Kendall, T. (2016). Predicting suicide following self-harm: Systematic review of risk factors and risk scales. British Journal Of Psychiatry, 209(4), 279-285. doi: 10.1192/bjp.bp.115.170050

Chan, A., Tetzlaff, J.M., Altman, D.G., Laupacis, A., Gotzsche, P.C., Krleza-Jeric, K., . . . Moher, D. (2013). SPIRIT 2013 statement: Defining standard protocol items for clinical trials.(Research and Reporting Methods)(Author abstract). Annals of Internal Medicine, 158(3), 200-207.

Chorpita, B.F., Yim, L., Moffitt, C., Umemoto, L.A., \& Francis, S.E. (2000). Assessment of symptoms of DSM-IV anxiety and depression in children: A revised child anxiety and depression scale. Behaviour Research and Therapy, 38(8), 835-855. doi: 10.1016/S00057967(99)00130-8

Crowell, S.E., Beauchaine, T.P., Hsiao, R.C., Vasilev, C.A., Yaptangco, M., Linehan, M.M., \& McCauley, E. (2012). Differentiating adolescent self-injury from adolescent depression: Possible implications for borderline personality development. Journal of Abnormal Child Psychology, 40(1), 45-57. doi: 10.1007/s10802-011-9578-3

De Silva, S., Parker, A., Purcell, R., Callahan, P., Liu, P., \& Hetrick, S. (2013). Mapping the evidence of prevention and intervention studies for suicidal and self-harming behaviors in young people. Crisis: The Journal of Crisis Intervention and Suicide Prevention, 34(4), 223-232. doi: 10.1027/0227-5910/a000190

Gratz, K., \& Roemer, L. (2004). Multidimensional assessment of emotion regulation and dysregulation: Development, factor structure, and initial validation of the difficulties in emotion regulation scale. Journal of Psychopathology and Behavioral Assessment, 26(1), 41-54. doi: 10.1023/B:JOBA.0000007455.08539.94

Ha, C., Sharp, C., Ensink, K., Fonagy, P., \& Cirino, P. (2013). The measurement of reflective function in adolescents with and without borderline traits. Journal of Adolescence, 36(6), 1215-1223. doi: 10.1016/j.adolescence.2013.09.008

Harb, G.C., Heimberg, R.G., Fresco, D.M., Schneier, F.R., \& Liebowitz, M.R. (2002). The psychometric properties of the interpersonal sensitivity measure in social anxiety disorder. Behaviour Research and Therapy, 40(8), 961-979. doi: 10.1016/S00057967(01)00125-5

Hawton, K., \& Harriss, L. (2007). Deliberate self-harm in young people: Characteristics and subsequent mortality in a 20 -year cohort of patients presenting to hospital. Journal of Clinical Psychiatry, 68(10), 1574-1583.

Hawton, K., \& Harriss, L. (2008). Deliberate Self-Harm by under-15-Year-Olds: Characteristics, Trends and Outcome. Journal of Child Psychology and Psychiatry, 49(4), 441-448. doi: 10.1111/j.1469-7610.2007.01852.x

Hawton, K., \& James, A. (2005). ABC of adolescence - Suicide and deliberate self harm in young people. British Medical Journal, 330(7496), 891-894.

Hawton, K., Saunders, K.E.A., \& O'Connor, R.C. (2012). Self-harm and suicide in adolescents. The Lancet, 379(9834), 2373-2382. doi: 10.1016/S0140-6736(12)60322-5

Hawton, K., Witt, K.G., Taylor Salisbury, T.L., Arensman, E., Gunnell, D., Townsend, E., van Heeringen, K., \& Hazell, P. (2015). Interventions for self-harm in children and adolescents. Cochrane Database of Systematic Reviews. Art.No.: CD012013

Karterud, S., \& Bateman, A. (2011). Manual for mentalization based psycho educational group therapy (MBT-I). Oslo: Gyldendal akademisk.

Mikolajczak, M., Petrides, K., \& Hurry, J. (2009). Adolescents choosing self-harm as an emotion regulation strategy: The protective role of trait emotional intelligence. British Journal of Clinical Psychology, 48(2), 181-193. doi: 10.1348/014466508X386027 
Ougrin, D., Tranah, T., Leigh, E., Taylor, L., \& Asarnow, J. (2012). Practitioner review: Self-harm in adolescents. Journal of Child Psychology and Psychiatry, 53(4), 337-350. doi: $10.1111 / \mathrm{j} .1469-7610.2012 .02525 . \mathrm{x}$

Ougrin, D., Tranah, T., Stahl, D., Moran, P., \& Asarnow, J.R. (2015). Therapeutic interventions for suicide attempts and self-harm in adolescents: Systematic review and meta-analysis. Journal of the American Academy of Child \& Adolescent Psychiatry, 54(2), 97-107.e2. doi: 10.1016/j.jaac.2014.10.009

Piqueras, J.A., Martín-Vivar, M., Sandin, B., San Luis, C., \& Pineda, D. (2017). The revised child anxiety and depression scale: A systematic review and reliability generalization meta-analysis. Journal of Affective Disorders, 218, 153-169. doi: 10.1016/j.jad.2017.04.022

Rossouw, Trudie I., \& Fonagy, Peter. (2012). Mentalization-based treatment for self-harm in adolescents: A randomized controlled trial. Journal of the American Academy of Child \& Adolescent Psychiatry, 51(12), 1304-1313.e3. doi: 10.1016/j.jaac.2012.09.018

Sharp, C., Steinberg, L., Temple, J., Newlin, E., \& Lejuez, Carl W. (2014). An 11-Item measure to assess borderline traits in adolescents: Refinement of the BPFSC using IRT. Personality Disorders: Theory, Research, and Treatment, 5(1), 70-78. doi: $10.1037 /$ per0000057

Van Oort, F. V. A., Greaves-Lord, K., Verhulst, F. C., Ormel, J., \& Huizink, A. C. (2009). The developmental course of anxiety symptoms during adolescence: The TRAILS study. Journal of Child Psychology and Psychiatry, 50(10), 1209-1217. doi: 10.1111/j.1469-7610.2009.02092.x

Vrouva, I., Fonagy, P., Fearon, P.R.M., \& Roussow, T. (2010). The risk-taking and self-harm inventory for adolescents: Development and psychometric evaluation. Psychological Assessment, 22(4), 852-865. doi: 10.1037/a0020583

Weinberg, A., \& Klonsky, E.D. (2009). Measurement of emotion dysregulation in adolescents. Psychological Assessment, 21(4), 616-621. doi: 10.1037/a0016669

World Health Organization. (2017). Adolescents: health risks and solutions. Retrieved from http://www.who.int/mediacentre/factsheets/fs345/en/. 